\title{
Vitamin E confers cytoprotective effects on cardiomyocytes under conditions of heat stress by increasing the expression of metallothionein
}

\author{
XIAOWU WANG, WENPENG DONG, BINBIN YUAN, YONGCHAO YANG, \\ DONGPENG YANG, XI LIN, CHANGFU CHEN and WEIDA ZHANG \\ Center of Cardiovascular Surgery, Guangzhou General Hospital of Guangzhou Military Command, \\ Guangzhou, Guangdong 510010, P.R. China
}

Received June 15, 2015; Accepted March 18, 2016

DOI: $10.3892 /$ ijmm.2016.2543

\begin{abstract}
Heat stress (HS) is commonly used to refer to the heat load that an individual is subjected to due to either metabolic heat, or environmental factors, including high temperatures and high humidity levels. HS has been reported to affect and even damage the functioning of various organs; overexposure to high temperatures and high humidity may lead to accidental deaths. It has been suggested that the cardiovascular system is primarily targeted by exposure to HS conditions; the HS-induced dysfunction of cardiomyocytes, which is characterized by mitochondrial dysfunction, may result in the development of cardiovascular diseases. The excessive production of reactive oxygen species (ROS) also participates in mitochondrial dysfunction. However, effective methods for the prevention and treatment of mitochondrial and cardiovascular dysfunction induced by exposure to HS are lacking. In the present study, we hypothesized that vitamin $\mathrm{E}$ (VE), an antioxidant, is capable of preventing oxidative stress and mitochondrial injury in cardiomyocytes induced by exposure to HS. The results revealed that pre-treatment with VE increased the expression of metallothionein (MT), which has previously been reported to confer cytoprotective effects, particularly on the cardiovascular system. Pre-treatment with VE restored mitochondrial function in cardiomyocytes under conditions of HS by increasing the expression of peroxisome proliferator-activated receptor- $\gamma$ coactivator- $1 \alpha$ (PGC- $1 \alpha$ ),
\end{abstract}

Correspondence to: Professor Weida Zhang, Center of Cardiovascular Surgery, Guangzhou General Hospital of Guangzhou Military Command, 111 Liuhua Road, Guangzhou, Guangdong 510010, P.R. China E-mail:weidazhanggz@163.com

Abbreviations: HS, heat stress; MT, metallothionein; VE, vitamin $\mathrm{E}$; ROS, reactive oxygen species; PGC- $1 \alpha$, peroxisome proliferatoractivated receptor-gamma coactivator-1 $\alpha$; NRF-1, nuclear respiratory factor 1; TFAM, mitochondrial transcription factor A; SOD, superoxide dismutase; GSH, glutathione; MDA, malondialdehyde; LDH, lactate dehydrogenase

Key words: heat stress, metallothionein, vitamin E, cardiomyocytes, cytoprotection nuclear respiratory factor 1 (NRF-1) and mitochondrial transcription factor A (TFAM), and by increasing adenosine triphosphate (ATP) levels. Furthermore, pre-treatment with VE decreased the production of ROS, which was induced by exposure to HS and thus exerted antioxidant effects. In addition, pre-treatment with VE attenuated oxidative stress induced by exposure to HS, as demonstrated by the increased levels of antioxidant enzymes [superoxide dismutase (SOD) and glutathione (GSH)], and by the decreased levels of markers of oxidative injury [malondialdehyde (MDA) and lactate dehydrogenase $(\mathrm{LDH})]$. Taken together, these findings suggest that pre-treatment with VE can prevent mitochondrial dysfunction and oxidative stress in cardiomyocytes induced by exposure to HS, by increasing the expression of MT.

\section{Introduction}

Increasing evidence has shown that heat stress (HS; namely, the heat overload induced by high temperatures and high humidity) negatively affects thermoregulation, limiting sweat evaporation and thus leading to high body temperatures and a series of heat stress responses (1). It has been suggested that the cardiovascular system is primarily targeted by exposure to HS and it is the most easily injured (2), which may result in the development of a series of heat-related illnesses, including heat cramps, heat syncope, heat exhaustion, heat stroke and even death (3). Mitochondria are abundantly distributed in cardiomyocytes due to the high energy demands of the heart (4), and mitochondria are vulnerable to various types of stress stimuli (3). The impairment of multiple aspects of mitochondrial function has been reported to be one of the principal events occuring in cardiomyocytes subjected to stress, including the increased permeability of the mitochondrial membrane, decreased membrane potential and the increased release of cytochrome $c$ and apoptosis-inducing factor (AIF) from the nucleus into the cytoplasm (5). Furthermore, previous research has indicated that HS reduces blood flow, which leads to a lower oxygen supply and cellular hypoxia; hypoxia in turn results in the excessive production of reactive oxygen species (ROS) and oxidative stress (6). ROS, including superoxide and hydrogen peroxide, are capable of destroying the structure and function of the cellular membrane, and of damaging the mitochondria, even- 
tually leading to cell death (7). Hence, targeting mitochondrial injury and the metabolism of ROS may confer cytoprotective effects on heat-stressed cardiomyocytes (exposed to high temperatures and humidity), and may thus provide a therapeutic strategy for cardiovascular diseases induced by exposure to HS.

To date, we have already elucidated one of the mechanisms responsible for the development of HS-related diseases; namely, the angiotensin II signaling pathway, which is involved in HS-induced oxidative stress and in the apoptosis of cardiomyocytes (8). Furthermore, we have proved in a previous study that a drug, geranylgeranylacetone, is effective at promoting the expression of heat shock protein 70 (Hsp70) (9). Hsp70 has been reported to confer effective cytoprotection against various types of stress stimuli (10). Metallothionein (MT) is also considered an endogenous cytoprotective molecule, mainly participating in the protection of the cardiovascular system (11). MT has also been reported to be involved in metal homeostasis and detoxification, radical scavenging and in maintaining the integrity of membrane structures (12). However, the protective effects exerted by MT on cardiomyocytes under conditions of HS remain to be elucidated. It is widely accepted that vitamin $\mathrm{E}$ (VE) is an effective antioxidant (13). In the present study, we hypothesized that VE may exert a synergistic effect with MT, both of which participate in the maintenance of mitochondrial function and ROS levels under conditions of HS.

The tropical marine climate of Southern China, which is usually hot, wet and rainy, results in excessive heat and humidity which may account for the high morbidity associated with cardiovascular diseases in this region. However, little is known regarding therapeutic approaches which confer cytoprotective effects on cardiomyocytes under conditions of HS. Thus, in the present study, we aimed to examine the effects of VE on cardiomyocytes under conditions of HS. For this purpose, mice were housed in an artificial environment in order to mimic conditions of HS. Pre-treatment with VE increased the expression of MT under conditions of HS. The restoration of mitochondrial function was indicated by the upregulation of peroxisome proliferator-activated receptor- $\gamma$ coactivator- $1 \alpha$ (PGC-1 $\alpha)$ and nuclear respiratory factor 1 (NRF-1), mitochondrial transcription factor A (TFAM), and increased adenosine triphosphate (ATP) levels. Moreover, conditions of HS increased the production of ROS and led to oxidative stress, and these effects were counteracted by pre-treatment with VE. The decrease in oxidative stress following treatment with $\mathrm{VE}$ was evidenced by the increased levels of antioxidant enzymes [superoxide dismutase (SOD) and glutathione (GSH)], and by the decreased levels of markers of oxidative injury [malondialdehyde (MDA) and lactate dehydrogenase (LDH)]. Taken together, these findings demonstrate that pre-treatment with VE confers cytoprotective effects on cardiomyocytes, possibly by increasing MT expression under conditions of HS. Our study provides evidence that VE may be used for the prevention and treatment of cardiovascular diseases induced by exposure to HS.

\section{Materials and methods}

Animals. Eight-week-old BALB/c mice, weighing 30-35 g, were purchased from the Laboratory Animal Center of Guangdong Province (Foshan, China). The mice were housed in a pathogenfree environment at a constant temperature of $20.0 \pm 2^{\circ} \mathrm{C}$ under a 12-h day and night cycle and fed a routine diet with free access to water. All animal experimental procedures were performed according to the guidelines of the National Health and Medical Research Council for the Care and Use of Animals for Experimental Purposes in China. This study was approved by the Ethics Committee of Guangzhou General Hospital of Guangzhou Military Command (Guangzhou, China). All efforts were made to minimize suffering and all the mice survived in this study without developing any infections. The mice were euthanized by an intraperitoneal injection of pentobarbital sodium (100 mg/kg, Merck KGaA, Darmstadt, Germany).

Establishment of the model of HS and experimental grouping. In order to mimic HS, a hot chamber was used to create an environment with a designated room temperature $\left(40.0 \pm 0.05^{\circ} \mathrm{C}\right)$ and relative humidity $(60 \pm 5 \%)$. Normal temperature and humidity $(\mathrm{NTH})$ conditions were designated as a room temperature of $24.0 \pm 1{ }^{\circ} \mathrm{C}$ and relative humidity of $45 \pm 5 \%$. A total of 40 mice was randomly allocated to 1 of the following 4 groups: i) 10 mice treated with the vehicle were housed under conditions of NTH; ii) 10 mice treated with VE were housed under conditions of NTH; iii) 10 mice treated with the vehicle were housed under conditions of HS for $4 \mathrm{~h}$ per day; and iv) 10 mice treated with VE were housed under conditions of HS for $4 \mathrm{~h}$ per day. VE (Novartis Co., Ltd., Tokyo, Japan) dissolved in ethyl alcohol was administered orally $(500 \mathrm{mg} / \mathrm{kg}$ ) using feeding needles, as previously reported (14), $2 \mathrm{~h}$ prior to the initiation of the experiment. The equivalent volume of ethyl alcohol was used as the vehicle, which was also administered orally using feeding needles. This experiment was conducted over a 4-week period.

Measurement of ATP levels. The mice were treated as described above and sacrificed under deep anesthesia. The mouse hearts were quickly excised and frozen in liquid nitrogen in preparation for subsequent use. The ATP levels in the heart tissues were measured using an ATP assay kit (Beyotime, Shanghai, China) according to the manufacturer's instructions. Briefly, heart tissues were subjected to ATP Detection Lysis buffer, followed by centrifugation at $13,000 \times \mathrm{g}$ for $5 \mathrm{~min}$, and the supernatant was added to the substrate solution. The relative light units were measured using a GloMax ${ }^{\mathrm{TM}} 96$ microplate luminometer (Promega, Madison, WI, USA). A standard curve was used to calculate the ATP concentration.

Ferric reducing/antioxidant power (FRAP) assay. A working FRAP solution was prepared by mixing 10 volumes of $300 \mathrm{mM}$ acetate buffer ( $\mathrm{pH} 3.6$ ) with 1 volume of $10 \mathrm{mM}$ tripyridyltriazine (TPTZ) solution and 1volume of $20 \mathrm{mM} \mathrm{FeCl}_{3}$ solution. TPTZ was dissolved in $40 \mathrm{mM}$ hydrochloric acid. Mouse cardiomyocytes were isolated as previouslsy described (15). Briefly, the BALB/c mice were sacrificed under deep anesthesia and their hearts were quickly excised. Within $3 \mathrm{~min}$, the heart tissues were cut into chunks, digested with collagenase type II (Worthington, Lakewood, NJ, USA) and protease type XIV (Sigma-Aldrich, St. Louis, MO, USA), and gently aspirated with a transfer pipette for facilitating the cell dissociation. The dissociated tissue explants were placed in minimal essential medium (MEM; HyClone, Logan, UT, USA) containing $12 \mathrm{mM} \mathrm{NaHCO} 3,10 \%$ fetal bovine serum (FBS; HyClone) and 
Table I. List of primer sequences used for RT-qPCR.

\begin{tabular}{lll}
\hline Gene & \multicolumn{1}{c}{ Sense } & \multicolumn{1}{c}{ Antisense } \\
\hline PGC-1 $\alpha$ & 5'-GATGTCAGTGACCTCGATGCA-3' & 5'-CAGCAAGTTGGCCTCATTTTC-3' \\
NRF-1 & 5'-CCACATTACAGGGCGGTGAA-3' & 5'-AGTGGCTCCCTGTTGCATCT-3' \\
TFAM & 5'-CGCCCTAGTAATATCGATCC-3' & 5'-ATGTTAATCGCTGGAATTGC-3' \\
MT & 5'-GTGTCCACTCCTGACCAGTATCCTT-3' & 5'-TCACAGCAGCCAGCATCTCTTCCAT-3' \\
GAPDH & 5'-AGGTCGGTGTGAACGGATTTG-3' & 5'-GGGGTCGTTGATGGCAACA-3' \\
\hline
\end{tabular}

PGC-1 $\alpha$, peroxisome proliferator-activated receptor $\gamma$ coactivator-1 $\alpha$; NRF-1, nuclear respiratory factor 1; TFAM, mitochondrial transcription factor A; MT, metallothionein.

$1 \%$ penicillin-streptomycin (HyClone) at $37^{\circ} \mathrm{C}$ with $5 \% \mathrm{CO}_{2}$ for cellular migration and confluence. The isolated cardiomyocytes were also cultured in MEM supplemented with $10 \%$ FBS and $1 \%$ penicillin-streptomycin. For the acquisition of samples, the cultured cardiomyocytes were lysed in RIPA buffer (Sigma-Aldrich) instead of the medium, followed by repetitive shaking on ice for $10 \mathrm{~min}$. The mixtures were collected as FRAP samples. For the acquisition of serum, the blood was drawn from heart artery before sacrifice, and allowed to stand for $1 \mathrm{~h}$ at $37^{\circ} \mathrm{C}$ for coagulation, followed by centrifugation at $3,000 \mathrm{x} \mathrm{g}$ for $10 \mathrm{~min}$. The supernatant was regarded as serum samples. The samples from both cardiomyocytes and serum were subjected to FRAP assay. Subsequently, $150 \mu \mathrm{l}$ of $\mathrm{FeSO}_{4}$ solution $(0,0.2,0.40 .6,0.8$ and $1.0 \mathrm{mM})$ was mixed with $4.5 \mathrm{ml}$ FRAP working solution for reaction at $37^{\circ} \mathrm{C}$ for $10 \mathrm{~min}$, and the absorbance at $593 \mathrm{~nm}$ was recorded for drawing a standard curve. Ten microliters of the samples were also mixed with FRAP working solution for reaction at $37^{\circ} \mathrm{C}$ for $10 \mathrm{~min}$, and the absorbance at $593 \mathrm{~nm}$ was recorded. The samples isolated from the mice housed under NTH conditions without VE pre-treatment served as the controls. The corresponding concentration of $\mathrm{FeSO}_{4}(\mu \mathrm{M})$ was set as the antioxidant potential, which was read from standard curve according to the absorbance of $\mathrm{FeSO}_{4}$ that was identical with samples.

Evaluation of ROS production. In order to evaluate ROS production, the mice were treated as described above for 4 weeks and sacrificed under deep anesthesia. The mouse hearts were rapidly excised and frozen in liquid nitrogen. The levels of ROS in the tissues were quantified using electron spin resonance spectroscopy with 4-hydroxy-2,2,6,6-tetramethylpiperidine-1-oxyl (4-hydroxy-TEMPO.7). All measurements were performed in 3 parallel runs.

Determination of oxidative stress. A biochemical analysis kit (Jiancheng Biotechnology Co., Nanjing, China) was used to measure the activity of SOD, GSH, MDA and LDH according to the manufacturer's instructions. Briefly, the heart tissues were washed in cold $0.9 \% \mathrm{NaCl}$ solution repetitively, to wipe out blood residue, followed by drying with filter paper and weighing. Subsequently, the heart tissues were transferred to a beaker, and $0.9 \% \mathrm{NaCl}$ solution was added again in a weight-to-volume ratio of 1:9. The heart tissues were then cut into sections and homogenized, followed by centrifugation at 2,000 $\mathrm{x}$ g for $15 \mathrm{~min}$. The supernatant was aspirated and subjected to respective analysis kits for SOD $(550 \mathrm{~nm})$, GSH $(420 \mathrm{~nm})$, MDA (532 nm) and LDH (450 nm) using a Luminometer (Promega).

Reverse transcription-quantitative polymerase chain reaction $(R T-q P C R)$. The left ventricular tissue was isolated along the interventricular septum. RNA was extracted from the left ventricular tissue using TRIzol reagent (Invitrogen, Carlsbad, CA, USA) according to the manufacturer's instructions. Total RNA $(5 \mu \mathrm{g})$ was reverse transcribed into cDNA using M-MLV reverse transcriptase (Clontech, Palo Alto, CA, USA) according to the manufacturer's instructions. The SsoFast EvaGreen Supermix (Bio-Rad, Hercules, CA, USA) was used in order to perform RT-qPCR and GAPDH served as the internal control. The following PCR conditions were used: $94^{\circ} \mathrm{C}$ for $4 \mathrm{~min}$; 35 cycles of $94^{\circ} \mathrm{C}$ for $20 \mathrm{sec}, 55^{\circ} \mathrm{C}$ for $30 \mathrm{sec}$, and $72^{\circ} \mathrm{C}$ for $20 \mathrm{sec}$ with $2 \mathrm{sec}$ for plate reading; and a melting curve from 65 to $95^{\circ} \mathrm{C}$. The experiments were performed independently at least 3 times. The fold expression was calculated using the $2^{-\Delta \Delta \mathrm{Ct}}$ method. The primer sequences are listed in Table I.

Western blot analysis. The proteins were extracted from the left ventricular tissue using RIPA buffer (Sigma-Aldrich) and the protein concentration was measured using the Bradford method. A total of $20 \mu \mathrm{g}$ protein was isolated by $12 \%$ SDS-PAGE followed by electroblotting onto a nitrocellulose (NC) membrane (Amersham, Little Chalfont, UK). Subsequently, non-specific binding was blocked with $2 \%$ skim milk in Tris-buffered saline (TBS) at room temperature for $1 \mathrm{~h}$. Subsequently, the NC membrane was incubated with primary antibodies (Santa Cruz Biotechnology, Santa Cruz, CA, USA) diluted in the blocking buffer overnight at $4^{\circ} \mathrm{C}$, including rabbit anti-MT (sc-11377), rabbit anti-PGC-1 $\alpha$ (sc-13067), goat anti-NRF-1 (sc-30911), rabbit anti-TFAM (sc-28200) and goat anti-GAPDH (sc-48166), which was followed by incubation with horseradish peroxidase (HRP)-conjugated secondary antibodies (Santa Cruz Biotechnology), including rabbit anti-goat IgG (sc-2768) and goat anti-rabbit IgG (sc-2004). The proteins were visualized using 4-chloro-1-naphthol (4-CN). Densitometric analysis was performed using Image Pro Plus 6.0 software (Media Cybernetics, Inc., Rockville, MD, USA).

Statistical analysis. All experiments were performed in triplicate and the data are presented as the means \pm standard deviation (SD). The statistical differences between 2 groups were determined using a Student's t-test, and were analyzed 

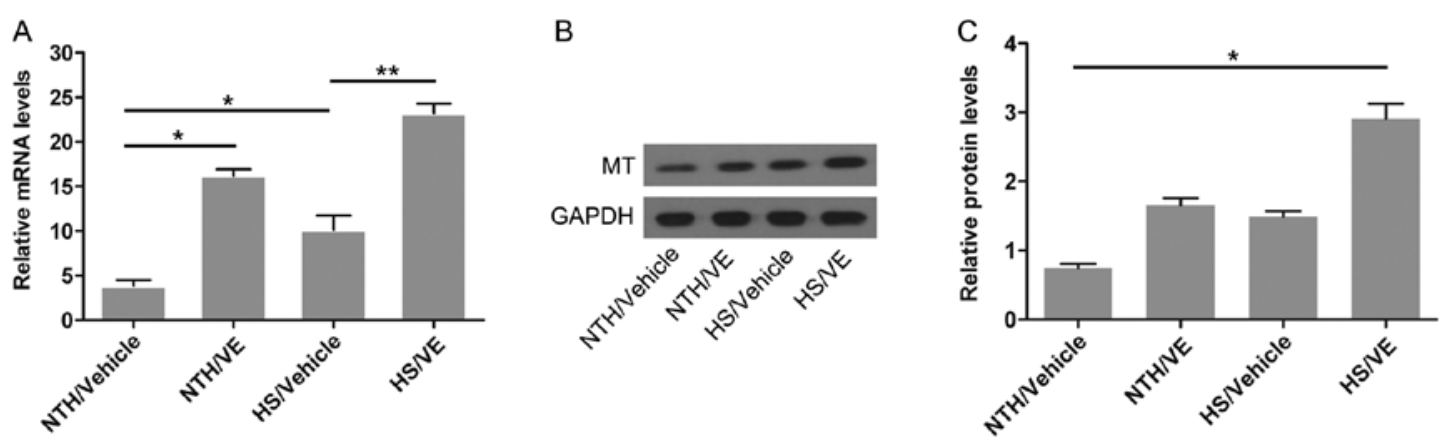

Figure 1. Effect of vitamin E (VE) on the mRNA and protein expression of metallothionein (MT). Determination of (A) mRNA levels and (B and C) protein levels of MT in mouse cardiomyoctes. Protein expression was analyzed using Image-Pro Plus 6.0 software and normalized to GAPDH. Each column represents the means $\pm \mathrm{SD} ; \mathrm{n}=3$ experiments; ${ }^{*} \mathrm{p}<0.05$ and ${ }^{* *} \mathrm{p}<0.01$. HS, heat stress; $\mathrm{NTH}$, normal temperature and humidity.

by one-way ANOVA within multiple groups. A p-value $<0.05$ was considered to indicate a statistically significant difference. All statistical analyses were performed using SPSS software version 11.5 (SPSS Inc., Chicago, IL, USA).

\section{Results}

Pre-treatment with VE increases the $m R N A$ and protein expression of MT in cardiomyocytes under conditions of HS. MT has been previously reported to play a cytoprotective role, particularly in cardiomyocytes $(16,17)$. Thus, in this study, to determine whether MT also participates in the stress response of cardiomyocytes exposed to high temperatures and humidity, the mRNA and protein levels of MT in mouse left ventricular tissue were measured by RT-qPCR and western blot analysis, respectively. As shown in Fig. 1A, the mRNA expression of MT was slightly increased ( $\mathrm{p}=0.0318 ; 16.2 \pm 0.71$-fold) by pretreatment with VE under conditions of NTH compared with the control group (NTH/vehicle; $3.9 \pm 0.65$-fold). In the absence of pre-treatment with VE, MT expression was also slightly increased $(\mathrm{p}=0.0219 ; 10.1 \pm 1.64$-fold $)$ in the cardiomyoctes under conditions of HS. Notably, pre-treatment with VE significantly upregulated the mRNA expression of MT ( $\mathrm{p}=0.0038$; $23.2 \pm 1.11$-fold) in the cardiomyocytes under conditions of HS compared with the NTH/vehicle group. The above-mentioned results suggested that VE promoted the transcription of the MT gene in the mouse cardiomyocytes, particularly under conditions of HS. As shown in Fig. 1B and C, pre-treatment with VE significantly increased the protein expression of MT under conditions of HS ( $p=0.0375 ; 2.9 \pm 0.22$-fold) compared with the other groups, implying that the translation of the MT gene was also enhanced by pre-treatment with VE in the mouse cardiomyocytes under conditions of HS.

Pre-treatment with VE restores mitochondrial function in cardiomyocytes under conditions of HS. The impairment of multiple aspects of mitochondrial function has been reported to be one of the principal events occuring in cardiomyocytes exposed to HS (3). An effective method of restoring mitochondrial function is urgently required. In this study, to determine whether VE promotes the recovery of mitochondrial function in cardiomyocytes under conditions of HS, the expression of genes involved in the regulation of mitochondrial function was measured in mouse left ventricular tissue by RT-qPCR and western blot analysis. The mRNA expression of PGC- $1 \alpha$, a transcriptional activating factor which participates in mitochondrial synthesis (18), was significantly reduced in the cardiomyocytes isolated from mice housed under conditions of HS ( $\mathrm{p}=0.0385)$; however, its expression was significantly increased by pre-treatment with VE ( $\mathrm{p}=0.0061$; Fig. 2A). The mRNA expression of NRF-1, a transcription factor regulating the transcription and replication of mitochondrial DNA (19), was decreased under conditions of HS ( $\mathrm{p}=0.0313$ ), suggesting the suppression of mitochondrial function; however, pre-treatment with VE significantly increased the expression of NRF-1 in the cardiomyocytes under conditions of HS ( $\mathrm{p}=0.0254$; Fig. 2B). The mRNA expression of TFAM, which plays a role in mitochondrial transcription and replication (20), was also significantly reduced in the cardiomyocytes under conditions of HS ( $\mathrm{p}=0.0246)$, and significantly increased by pre-treatment with VE ( $\mathrm{p}=0.0369$; Fig. $2 \mathrm{C})$. The protein expression of these 3 genes also confirmed the above-mentioned alterations (Fig. 2A-C). These data suggest that pre-treatment with $\mathrm{VE}$ restores mitochondrial function in cardiomyocytes under conditions of HS.

Pre-treatment with VE increases ATP levels in mouse heart tissue under conditions of HS. To further confirm the effects of VE on mitochondrial function in mouse heart tissue under conditions of HS, the levels of ATP, an indicator of mitochondrial function, were measured using an ATP assay kit. As shown in Fig. 3, the ATP levels were significantly reduced in the mouse heart tissue under conditions of HS ( $p=0.0273$ ), indicating that the exposure to HS impaired mitochondrial function. By contrast, pre-treatment with VE increased the ATP levels in the mouse heart tissue under conditions of HS ( $p=0.0063$ ), which demonstrated that the restoration of mitochondrial function had occurred.

Antioxidant capacity is reduced under conditions of $H S$, but these effects are reversed by pre-treatment with VE. Oxidative stress is another event induced by exposure to HS and antioxidative capacity is an indicator of oxidative stress. In order to determine the effects of pre-treatment with VE on the antioxidant capacity in serum and cardiomyocytes obtained from mice exposed to conditions of HS, a FRAP assay was 

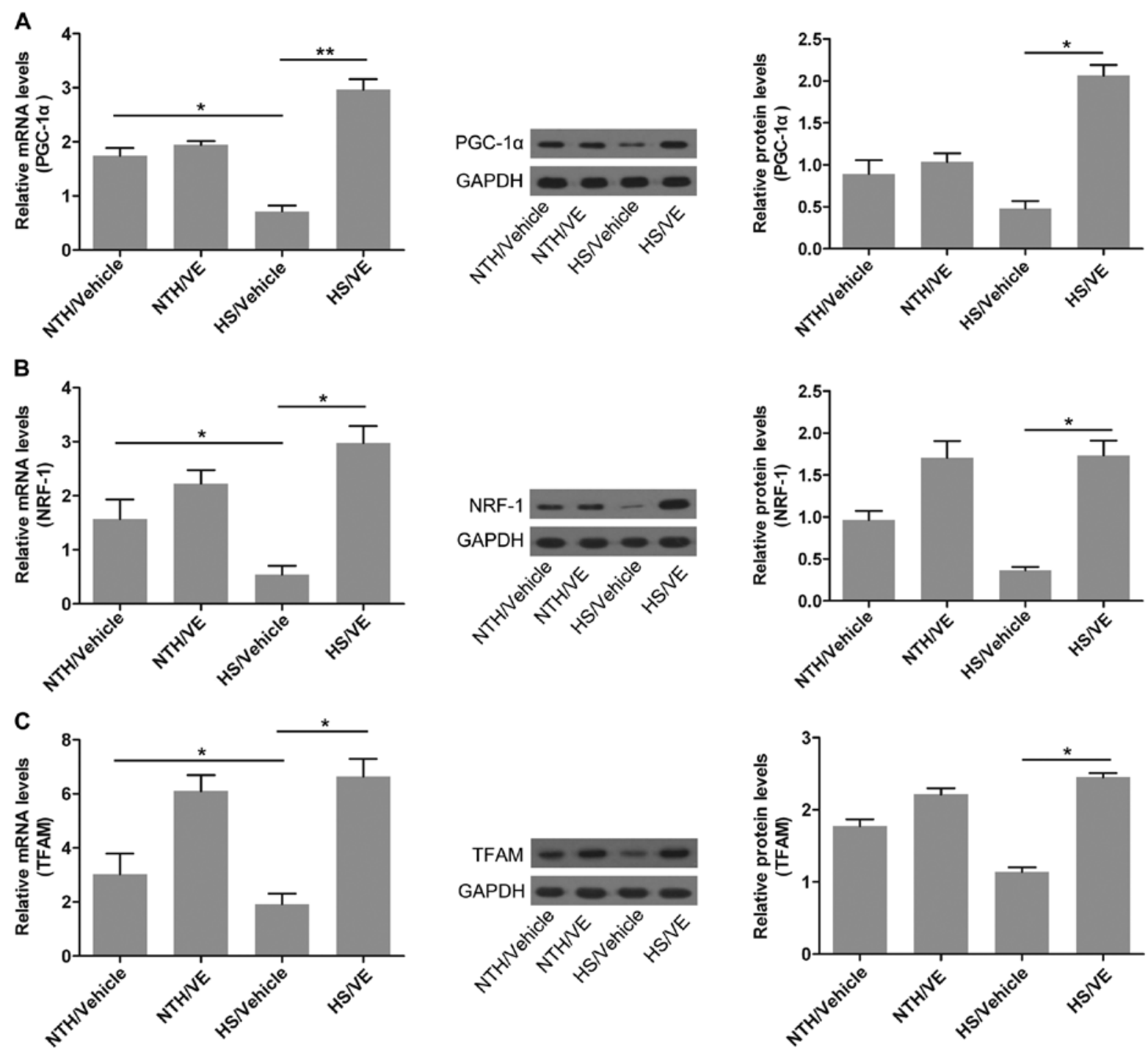

Figure 2. Effect of vitamin E (VE) on the mRNA and protein expression levels of genes involved in the regulation of mitochondrial functions. RT-qPCR and western blot analysis were performed to determine the mRNA and the protein levels, respectively. Protein expression was analyzed using Image-Pro Plus 6.0 software and normalized to GAPDH. (A) Peroxisome proliferator-activated receptor- $\gamma$ coactivator-1 $\alpha$ (PGC-1 $\alpha$ ) expression. (B) Nuclear respiratory factor 1 (NRF-1) expression. (C) Mitochondrial transcription factor A (TFAM) expression. Each column represents the means \pm SD; $n=3$ experiments; ${ }^{*} \mathrm{p}<0.05$ and ${ }^{* *} \mathrm{p}<0.01$. HS, heat stress; $\mathrm{NTH}$, normal temperature and humidity.

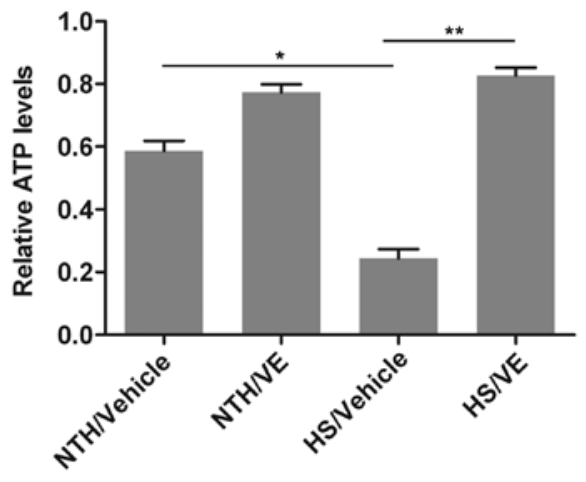

Figure 3. Analysis of ATP levels in mouse heart tissues. The relative ATP level was represented by fold changes quantified by comparing absorbance values using a standard curve. Each column represents the means $\pm \mathrm{SD} ; \mathrm{n}=3$ per group; ${ }^{*} \mathrm{p}<0.05$ and ${ }^{* *} \mathrm{p}<0.01$. HHS, heat stress; NTH, normal temperature and humidity.

performed. The results revealed that the antioxidant capacity was significantly decreased under conditions of HS compared with normal conditions (the NTH/vehicle group; $\mathrm{p}=0.0517$ in serum and 0.0580 in cardiomyocytes, respectively; Fig. 4), indicating that HS conditions promote oxidative stress. However, pre-treatment with VE significantly increased the antioxidant capacity in both the serum and the cardiomyocytes $(\mathrm{p}=0.0171$ in serum and 0.0159 in cardiomyocytes, respectively; Fig. 4), implying that VE may attenuate oxidative stress induced by exposure to HS.

Pre-treatment with VE decreases the production of ROS induced by exposure to HS. The level of ROS is another index of oxidative stress. The decreased antioxidant capacity may be attributed to the accumulation of ROS. In this study, to examine the effects of pre-treatment with VE on the production of ROS, the ROS levels in the mouse heart tissues were measured using electron spin resonance spectroscopy. As shown in Fig. 5, compared with the NTH/vehicle group, HS induced a significant increase in the production of ROS $(p=0.0042)$. Moreover, the pre-treatment with VE significantly decreased the produc- 


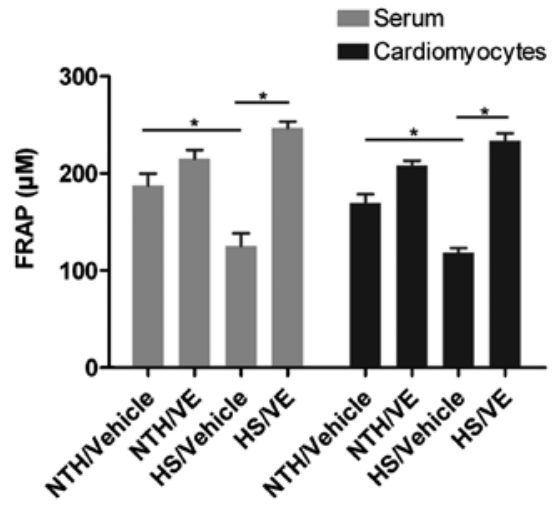

Figure 4. Determination of the antioxidant capacity in serum and cardiomyocytes. A ferric reducing/antioxidant power (FRAP) assay was performed according to standard protocols. Each column represents the means $\pm \mathrm{SD} ; \mathrm{n}=3$ experiments; " $\mathrm{p}<0.05$. HS, heat stress; NTH, normal temperature and humidity.

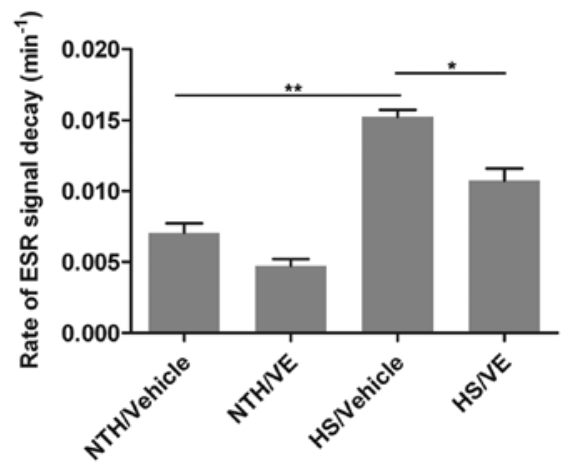

Figure 5. Evaluation of reactive oxygen species (ROS) levels in heart tissues. Electron spin resonance (ESR) analysis of 4-hydroxy-TEMPO.7 signal decay in the mouse hearts was performed. Each column represents the means $\pm \mathrm{SD}$; $\mathrm{n}=3$ experiments; ${ }^{*} \mathrm{p}<0.05$ and ${ }^{* *} \mathrm{p}<0.01$. HS, heat stress; NTH, normal temperature and humidity.
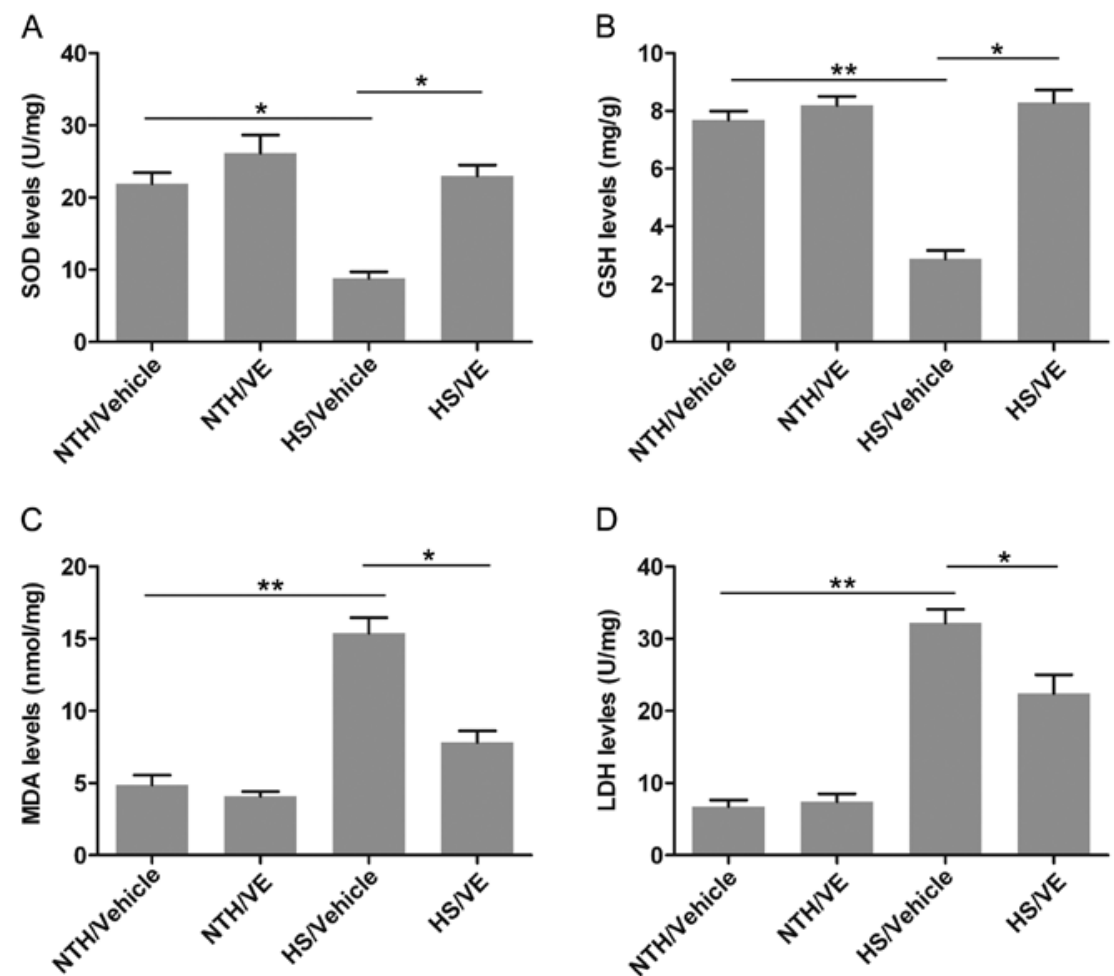

Figure 6. Determination of the levels of superoxide dismutase (SOD), glutathione (GSH), malondialdehyde (MDA) and lactate dehydrogenase (LDH) in cardiomyocytes. SOD and GSH are antioxidant enzymes; MDA and LDH are markers of oxidative injury. (A) SOD levels were determined at $500 \mathrm{~nm}$ and the values are expressed as U/mg protein. (B) GSH levels were detected at $420 \mathrm{~nm}$ and the data are shown as $\mathrm{mg} / \mathrm{g}$ protein. (C) MDA levels were measured at $532 \mathrm{~nm}$ and the results are presented as $\mathrm{nmol} / \mathrm{mg}$ protein. (D) LDH levels were measured at $500 \mathrm{~nm}$ and the values are expressed as $\mathrm{U} / \mathrm{mg}$ protein. Each column represents the means \pm SD; $n=3$ experiments; ${ }^{p} p<0.05$ and ${ }^{* *} \mathrm{p}<0.01$. HS, heat stress; NTH, normal temperature and humidity.

tion of ROS (p=0.0417; Fig. 5) compared with the HS/vehicle group. These results suggest that pre-treatment with VE reduces the production of ROS.

Pre-treatment with VE attenuates oxidative stress induced by exposure to $H S$. In order to examine the extent of oxidative stress induced by exposure to HS more specifically, we examined the effects of VE on the levels of antioxidant enzymes and markers of oxidative injury, namely SOD, GSH, MDA and LDH. The level of SOD, a powerful antioxidant which targets ROS, was significantly decreased under conditions of HS ( $\mathrm{p}=0.0134$; Fig. 6A); however, pre-treatment with VE significantly increased SOD levels $(p=0.0259)$. Similarly, the level of $\mathrm{GSH}$, a common antioxidant, was also significantly reduced in the HS/vehicle group ( $\mathrm{p}=0.0028$; Fig. $6 \mathrm{~B}$ ), whereas pre-treatment with VE significantly increased the GSH level $(\mathrm{p}=0.0147)$. The level MDA, an important marker of lipid peroxidation associated with oxidative stress, was significantly increased under conditions of HS compared with the NTH/vehicle group ( $\mathrm{p}=0.0034$; Fig. 6C); this effect was inhibited by pre-treatment with VE $(\mathrm{p}=0.0267)$. We also observed that exposure to HS significantly increased the release of $\mathrm{LDH}(\mathrm{p}=0.0043$; Fig. $6 \mathrm{D})$, whereas pre-treatment 
with VE reduced the LDH level $(\mathrm{p}=0.0412)$. Taken together, these results demonstrate that the oxidative stress induced by exposure to HS may be attenuated by pre-treatment with VE.

\section{Discussion}

In the present study, we demonstrate that pre-treatment with VE can prevent mitochondrial dysfunction and oxidative stress in cardiomyocytes induced by exposure to HS, possibly by increasing the expression of MT. Mitochondrial dysfunction and oxidative stress are the two principal events induced by exposure to HS $(21,22)$, which mainly target the cardiovascular system $(2,23)$. However, effective methods for the prevention and treatment of cardiovascular diseases caused by exposure to HS are lacking. This study provides evidence that pre-treatment with VE has potential therapeutic applications as it is capable of counteracting the effects of exposure to HS. However, the application of VE in the prevention of HS warrants further investigation.

In our previous studies, one of the underlying mechanisms responsible for heat stress (due to high temperatures and humidity), as well as a potential drug capable of protecting cardiomyocytes from HS were explored. More specifically, HS-induced oxidative stress and the apoptosis of cardiomyocytes were attributed to the renin-angiotensin system (8). Subsequently, it was proven that geranylgeranylacetone suppressed the apoptosis of cardiomyocytes exposed to HS, mainly due to the enhanced expression of Hsp70; however, it had no effects on oxidative stress (9). Cell apoptosis is the third event induced by HS (24), in addition to mitochondrial dysfunction and oxidative stress mentioned in the present study. In fact, mitochondrial dysfunction consists of a series of events, including the release of cytochrome $c$ and AIF from the mitochondrial nucleus into the cytoplasm, which leads to the insufficient supply of energy and subsequent cellular apoptosis (25). This process is termed mitochondria-mediated cell apoptosis. Hence, mitochondrial dysfunction and cell apoptosis are closely interrelated. Decreased cell survival induced by cell apoptosis has also been demonstrated to contribute to HS-induced cardiovascular diseases.

Currently, it is widely accepted that ROS are inter- and intra-cellular second messengers, participating in intracellular signal transmission $(26,27)$. However, the excessive production of ROS may exert negative effects, leading to oxidative stress, gene silencing, cellular dysfunction and ultimately, apoptosis or necrosis. ROS are a type of oxidant found in organisms. Hence, a balance between oxidation and antioxidation is crucial for normal metabolism, cellular function, as well as correct responses to various types of stimuli (28). Above all, the levels of antioxidants within cells, the cytomembrane, and the cytoplasm may be increased and mobilized to neutralize the excessive formation of ROS $(29,30)$. It has been previously reported that VE is an effective antioxidant; Shirpoor et al investigated the role of VE in diabetes-induced oxidative stress, and found that as an antioxidant, VE exerts a potent protective effect (31). In addition, Takahashi et al reported that VE supplementation attenuated oxidative stress in postmenopausal women (32). Moreover, in the study by Sakr et al, VE was proven to effectively attenuate oxidative stress in a rat model of hypoxia (33). However, the effects of VE on cardiovascular diseases induced by exposure to HS are not yet fully understood. In this study, our results suggest that exposure to HS may induce oxidative stress, as indicated by the overproduction of ROS and the decreased antioxidant capacity. Pre-treatment with VE was also demonstrated to attenuate oxidative stress induced by exposure to HS.

MT belongs to a group of intracellular, non-enzymatic proteins $(6-7 \mathrm{kDa})$ that is distinguished among different species (11), and it is characterized by the binding of metal ions, such as $\mathrm{Zn}^{2+}, \mathrm{Cd}^{2+}, \mathrm{Cu}^{2+}$ and $\mathrm{Hg}^{2+}$ (34). Ubiquitously expressed MT has been reported to participate in a number of regulatory activities in both normal and cancer cells, such as in the homeostasis of zinc ions, the detoxification of heavy metals, proliferation, apoptosis and cytoprotection against oxidative damage (35-39). In the present study, the expression of MT was increased by pre-treatment with VE, suggesting that MT participates in the protection of mouse cardiomyocytes against HS.

In conclusion, in this study, we demonstrated that the oral administration of VE increased the expression of MT, which was associated with the restoration of mitochondrial function and reduced oxidative stress. Thus, we provide evidence for the possible application of VE in the prevention and treatment of cardiomyocytes under conditions of HS. However, further investigations are warranted in order to provide more concrete evidence that the increased expression of MT directly contributes to the cytoprotective effects of $\mathrm{VE}$, as well as to elucidate the precise mechanisms responsible for these effects.

\section{Acknowledgements}

This study was supported by grants from the National Natural Science Foundation of China (81200633) and the China Postdoctoral Science Foundation (2013M532160).

\section{References}

1. Bouchama A and Knochel JP: Heat stroke. N Engl J Med 346: 1978-1988, 2002

2. Chien KR: Genomic circuits and the integrative biology of cardiac diseases. Nature 407: 227-232, 2000.

3. Qian L, Song X, Ren H, Gong J and Cheng S: Mitochondrial mechanism of heat stress-induced injury in rat cardiomyocyte. Cell Stress Chaperones 9: 281-293, 2004.

4. Carreira RS, Lee P and Gottlieb RA: Mitochondrial therapeutics for cardioprotection. Curr Pharm Des 17: 2017-2035, 2011.

5. Kyrychenko V, Poláková E, Janíček R and Shirokova N: Mitochondrial dysfunctions during progression of dystrophic cardiomyopathy. Cell Calcium 58: 186-195, 2015.

6. Hall DM, Buettner GR, Oberley LW, Xu L, Matthes RD and Gisolfi CV: Mechanisms of circulatory and intestinal barrier dysfunction during whole body hyperthermia. Am J Physiol Heart Circ Physiol 280: H509-H521, 2001.

7. Ray PD, Huang BW and Tsuji Y: Reactive oxygen species (ROS) homeostasis and redox regulation in cellular signaling. Cell Signal 24: 981-990, 2012.

8. Wang X, Yuan B, Dong W, Yang B, Yang Y, Lin X and Gong G: Humid heat exposure induced oxidative stress and apoptosis in cardiomyocytes through the angiotensin II signaling pathway. Heart Vessels 30: 396-405, 2015.

9. Wang X, Yuan B, Dong W, Yang B, Yang Y, Lin X and Gong G: Induction of heat-shock protein 70 expression by geranylgeranylacetone shows cytoprotective effects in cardiomyocytes of mice under humid heat stress. PLoS One 9: e93536, 2014.

10. Jäättelä M, Wissing D, Kokholm K, Kallunki T and Egeblad M: Hsp70 exerts its anti-apoptotic function downstream of caspase-3-like proteases. EMBO J 17: 6124-6134, 1998. 
11. Coyle P, Philcox JC, Carey LC and Rofe AM: Metallothionein: the multipurpose protein. Cell Mol Life Sci 59: 627-647, 2002.

12. Fukase Y, Tsugami H, Nakamura Y, Ohba K and Ohta H: The role of metallothionein and metal transporter on cadmium transport from mother to fetus. Yakugaku Zasshi 134: 801-804, 2014 (In Japanese).

13. Zhao XL, Li YK, Cao SJ, Hu JH, Wang WH, Hao RJ, Gui LS and Zan LS: Protective effects of ascorbic acid and vitamin $\mathrm{E}$ on antioxidant enzyme activity of freeze-thawed semen of Qinchuan bulls. Genet Mol Res 14: 2572-2581, 2015.

14. Peng L: Mice brain tissue injury induced by diisononyl phthalate exposure and the protective application of vitamin $\mathrm{E}$. J Biochem Mol Toxicol 29: 311-320, 2015.

15. Das A, Xi L and Kukreja RC: Phosphodiesterase-5 inhibitor sildenafil preconditions adult cardiac myocytes against necrosis and apoptosis. Essential role of nitric oxide signaling. J Biol Chem 280: 12944-12955, 2005.

16. Ye G, Metreveli NS, Ren J and Epstein PN: Metallothionein prevents diabetes-induced deficits in cardiomyocytes by inhibiting reactive oxygen species production. Diabetes 52: 777-783, 2003 .

17. Wang GW, Klein JB and Kang YJ: Metallothionein inhibits doxorubicin-induced mitochondrial cytochrome $\mathrm{c}$ release and caspase-3 activation in cardiomyocytes. J Pharmacol Exp Ther 298: 461-468, 2001.

18. Liang $\mathrm{H}$ and Ward WF: PGC-1alpha: a key regulator of energy metabolism. Adv Physiol Educ 30: 145-151, 2006.

19. Gleyzer N, Vercauteren K and Scarpulla RC: Control of mitochondrial transcription specificity factors (TFB1M and TFB2M) by nuclear respiratory factors (NRF-1 and NRF-2) and PGC-1 family coactivators. Mol Cell Biol 25: 1354-1366, 2005.

20. Ikeda M, Ide T, Fujino T, Arai S, Saku K, Kakino T, Tyynismaa H, Yamasaki T, Yamada K, Kang D, et al: Overexpression of TFAM or twinkle increases mtDNA copy number and facilitates cardioprotection associated with limited mitochondrial oxidative stress. PLoS One 10: e0119687, 2015.

21. Slimen IB, Najar T, Ghram A, Dabbebi H, Ben Mrad M and Abdrabbah M: Reactive oxygen species, heat stress and oxidativeinduced mitochondrial damage. A review. Int J Hyperthermia 30: 513-523, 2014.

22. Huang C, Jiao H, Song Z, Zhao J, Wang X and Lin H: Heat stress impairs mitochondria functions and induces oxidative injury in broiler chickens. J Anim Sci 93: 2144-2153, 2015.

23. Steenland K: Epidemiology of occupation and coronary heart disease: research agenda. Am J Ind Med 30: 495-499, 1996.

24. Havenith G, Luttikholt VG and Vrijkotte TG: The relative influence of body characteristics on humid heat stress response. Eur J Appl Physiol Occup Physiol 70: 270-279, 1995.

25. Lane RK, Hilsabeck T and Rea SL: The role of mitochondrial dysfunction in age-related diseases. Biochim Biophys Acta 1847: $1387-1400,2015$.
26. San Martín A, Du P, Dikalova A, Lassègue B, Aleman M, Góngora MC, Brown K, Joseph G, Harrison DG, Taylor WR, et al: Reactive oxygen species-selective regulation of aortic inflammatory gene expression in Type 2 diabetes. Am J Physiol Heart Circ Physiol 292: H2073-H2082, 2007.

27. Lassègue $B$ and Griendling KK: Reactive oxygen species in hypertension; An update. Am J Hypertens 17: 852-860, 2004.

28. Nordberg J and Arnér ES: Reactive oxygen species, antioxidants, and the mammalian thioredoxin system. Free Radic Biol Med 31: 1287-1312, 2001.

29. Halliwell B and Cross CE: Oxygen-derived species: their relation to human disease and environmental stress. Environ Health Perspect 102 (Suppl 10): 5-12, 1994.

30. Davies KJ: Oxidative stress: the paradox of aerobic life. Biochem Soc Symp 61: 1-31, 1995.

31. Shirpoor A, Norouzi L, Nemati S and Khadem Ansari MH: Protective effect of vitamin $\mathrm{E}$ against diabetes-induced oxidized LDL and aorta cell wall proliferation in rat. Iran Biomed J 19: 117-123, 2015.

32. Takahashi M, Miyashita M, Park JH, Kawanishi N, Bae SR, Nakamura Y, Sakamoto S and Suzuki K: Low-volume exercise training and vitamin E supplementation attenuates oxidative stress in postmenopausal women. J Nutr Sci Vitaminol (Tokyo) 59: 375-383, 2013.

33. Sakr HF, Abbas AM and El Samanoudy AZ: Effect of vitamin E on cerebral cortical oxidative stress and brain-derived neurotrophic factor gene expression induced by hypoxia and exercise in rats. J Physiol Pharmacol 66: 191-202, 2015.

34. Dziegiel P: Expression of metallothioneins in tumor cells. Pol J Pathol 55: 3-12, 2004.

35. Dziegiel P, Salwa-Zurawska W, Zurawski J, Wojnar A and Zabel M: Prognostic significance of augmented metallothionein (MT) expression correlated with $\mathrm{Ki}-67$ antigen expression in selected soft tissue sarcomas. Histol Histopathol 20: 83-89, 2005.

36. Pula B, Domoslawski P, Podhorska-Okolow M and Dziegiel P: Role of metallothioneins in benign and malignant thyroid lesions. Thyroid Res 5: 26, 2012.

37. Surowiak P, Matkowski R, Materna V, Györffy B, Wojnar A, Pudelko M, Dziegiel P, Kornafel J and Zabel M: Elevated metallothionein (MT) expression in invasive ductal breast cancers predicts tamoxifen resistance. Histol Histopathol 20: 1037-1044, 2005

38. Thirumoorthy N, Shyam Sunder A, Manisenthil Kumar K, Senthil Kumar M, Ganesh G and Chatterjee M: A review of metallothionein isoforms and their role in pathophysiology. World J Surg Oncol 9: 54, 2011.

39. Wojnar A, Pula B, Piotrowska A, Jethon A, Kujawa K, Kobierzycki C, Rys J, Podhorska-Okolow M and Dziegiel P: Correlation of intensity of MT-I/II expression with Ki-67 and MCM-2 proteins in invasive ductal breast carcinoma. Anticancer Res 31: 3027-3033, 2011. 